\title{
Nature et monde naturel dans la pensée de Jan Patočka
}

Ovidiu Stanciu

\section{(2) OpenEdition}

1 Journals

Édition électronique

URL : http://journals.openedition.org/alter/576

DOI : $10.4000 /$ alter.576

ISSN : 2558-7927

Éditeur :

Association ALTER, Archives Husserl (CNRS-UMR 8547)

\section{Édition imprimée}

Date de publication : 31 décembre 2018

Pagination : 47-64

ISBN : 978-2-9550449-4-0

ISSN : $1249-8947$

\section{Référence électronique}

Ovidiu Stanciu, « Nature et monde naturel dans la pensée de Jan Patočka », Alter [En ligne], 26 | 2018, mis en ligne le 31 décembre 2019, consulté le 24 mars 2020. URL : http://journals.openedition.org/ alter/576 ; DOI : https://doi.org/10.4000/alter.576 


\section{NATURE ET MONDE NATUREL DANS LA PENSÉE DE JAN PATOČKA}

Ovidiu Stanciu

Il y a du vrai dans la vieille Naturphilosophie dont l'erreur fut essentiellement de prétendre remplacer la physique mécaniste objective, alors que son motif propre était ailleurs, dans le fait qu'il y a d'autres manières d'accéder à la nature, des manières plus originaires que la démarche objectivante et idéalisante des sciences mathématiques de la nature ${ }^{1}$.

Le défi majeur qu'une enquête phénoménologique doit relever dès lors qu'elle dirige son regard thématique sur la question de la nature réside dans la circonscription du lieu théorique qu'une telle analyse doit occuper. La tâche de fournir une élucidation phénoménologique de la nature équivaut-elle au projet de restituer les articulations du domaine de sens au sein duquel évoluent les sciences mathématiques de la nature ? Afin de cerner le propre de la nature suffit-il de spécifier les contours et d'examiner les partages qui structurent cette région ? Est-ce que la nature désigne un champ d'objets quantifiables et mesurables, qui supporte une transcription en langage mathématique ou encore - selon la caractérisation de Whitehead - un domaine autarcique, un système fermé en lui-même et fermé à l'esprit ${ }^{2}$ ? Depuis Husserl, la phénoménologie a remis en cause l'autarcie proclamée de la nature et a mis en évidence sa dépendance à l'égard d'un monde déjà articulé. La nature est le corrélat d'un processus d'idéalisation, de sorte qu'elle devient, dans la Krisis, un « pôle infiniment éloigné » visé

\footnotetext{
${ }^{1}$ J. Patočka, «Leçons sur la corporéité » in Papiers phénoménologiques (désormais PP), trad. E. Abrams, Grenoble, Jérôme Millon, 1995, p. 104.

${ }^{2}$ Cf. A. N. Whitehead, Le concept de nature, trad. J. Douchement, Paris, Vrin, 1998, p. 10.
} 
par «une infinité de théories »3. Pourtant, on peut se demander si l'entreprise consistant à conjurer les dangers du naturalisme - c'est-àdire du projet de produire une pensée de la nature qui fait abstraction de la pensée comme telle - ne conduit pas à vider le concept de nature de toute sa teneur. Car dès lors que l'on fait apparaître que la «nature en soi » n'est que le corrélat d'un projet universel d'objectivation, ne doit-on pas admettre que «dans les sciences mathématiques de la nature, la nature n'est pas ce qui se montre soi-même, [qu']elle n'est pas phénomène, mais objet de constructions et d'expériences $\gg^{4}$ ? Envisagée selon cette optique, la nature semble dissoute dans le projet de sens qui lui accorde, tout d'abord, la possibilité d'apparaître, et s'avère être une abstraction idéalisante dépourvue de toute phénoménalité propre.

Devant les difficultés qu'une telle approche suscite, la tentation est grande d'abandonner cette chaîne multiple de médiations (expérimentales et fondationnelles) où l'appréhension de la nature est constamment (et constitutivement) différée et de s'en remettre à l'expérience immédiate que nous en faisons, à la manière dont elle se découvre dans notre commerce courant avec les choses. Les différentes manières de me frayer un chemin à travers le monde impliquent à chaque fois une prise en vue - fût-elle latérale ou périphérique - de la nature : «Dans les voies, routes, ponts, constructions, la nature se trouve découverte dans une orientation déterminée par la préoccupation $»^{5}$. Chacune des choses que nous utilisons, chacun des outils que nous employons contiennent des renvois implicites à l'étoffe dont ils sont faits, au bois dans lequel ils ont été taillés. En vaquant à nos besognes ou en laissant nos pas errer librement sur des chemins creux, nous sommes à chaque fois au milieu de la nature. Pourtant, ce que le comportement quotidien découvre ainsi c'est la nature-ambiante (Umweltnatur), une nature domestiquée et déjà insérée dans un circuit mondain. Découpée et ordonnée, pliée à nos besoins, la nature tend à se dissimuler comme dimension autonome et à n'apparaître que sous les traits d'une « réserve » dans laquelle on peut puiser les «ressources » nécessaires à l'aménagement de notre monde.

Si la teneur propre de la nature est oblitérée lorsqu' elle est envisagée comme étant d'emblée ajustée à nos pratiques et à nos prises, nous

\footnotetext{
${ }^{3}$ E. Husserl, Die Krisis der europäischen Wissenschaften und die transzendentale Phänomenologie, Hua VI, La Haye, Martinus Nijhoff, 1954, p. 41 ; trad. G. Granel, Paris, Gallimard, 1976, p. 49.

${ }^{4}$ J. Patočka, Essais hérétiques sur la philosophie de l'histoire, trad. E. Abrams, Paris, Verdier, 1999, p. 96.

${ }_{5}^{5}$ M. Heidegger, Sein und Zeit, GA 2, F.-W. von Herrmann (éd.), Francfort-sur-le-Main, Klostermann, 1977, p. 71 ; Être et temps, trad. E. Martineau, Paris, Authentica, 1985, p. 75-76.
} 
pouvons espérer recueillir sa manifestation exemplaire en la laissant se déployer à partir d'elle-même et en nous contenant d'accompagner du regard la propension inscrite en son sein. Saisie selon cette optique, la nature désigne la force générative qui fait que des êtres poussent et s'épanouissent spontanément, la puissance créatrice et aveugle qui jette l'étant dans la lumière et l'amène à une déterminité. En tant qu'elle nomme la source de tout jaillissement ontique, la nature-physis apparaît comme un visage de l'originaire ${ }^{6}$. Il n' est peut-être pas anodin que lorsqu'on a essayé, en phénoménologie, de faire intervenir un concept de nature qui ne doit rien à la science mathématisée ou à l'usage instrumentalisant, on a souvent fait appel au concept de physis : il y aurait comme un partage entre, d'un côté, la platitude d'une nature «naturaliste » ou " aménagée » et, de l'autre, l'impénétrabilité et l'exorbitance de la physis "grecque ». Pourtant, en dépit des lettres de noblesses que ce concept possède, on pourrait se demander si une nature excédentaire, porteuse et dépositaire de tout sens, n'a pas davantage l'allure d'un principe que celle d'un phénomène. Si la distinction entre ce qui doit son être à l'activité humaine - fût-il de l'ordre de l'artificiel ou du conventionnel - et ce qui surgit spontanément, ce qui pousse tout seul, a une base phénoménale indéniable, il n'est pas certain que la puissance de prolifération mondaine peut s'offrir, comme telle, dans une expérience. Il se peut que ce soit précisément son caractère élémentaire qui barre la voie à toute appréhension, qu'elle se dérobe à toute prise en vertu de son originarité même.

Ces développements nous permettent de définir les réquisits théoriques auxquels une pensée phénoménologique de la nature doit satisfaire. En effet, octroyer une place à la nature à l'intérieur de la démarche phénoménologique impose de mettre entre parenthèses (quitte, éventuellement, à les retrouver par la suite) les trois significations que nous venons d'exposer : la nature objectivée et appauvrie, tenue à distance par le dispositif des sciences de la nature ; la nature ajustée à l'humain, immédiatement instrumentalisée par la préoccupation quotidienne ; la nature excédentaire, entendue comme puissance ontogénétique et comme figure de l'élémentaire. Il faut donc que la nature apparaisse à même le monde sans pour autant se dissoudre

\footnotetext{
${ }^{6}$ La plus radicale prise de position assumant cette orientation au sein de la philosophie d'orientation phénoménologique appartient à M. Dufrenne. Cf. entre autres Pour l'homme, Paris, Seuil, 1968, p. 21 : «ne faut-il pas conférer à l'être la densité et le dynamisme d'une Nature, comme de la plante qui s'épanouit dans la lumière parce qu'elle est autre chose que la lumière ? [...] Cette plénitude infinie ne fait-elle pas défaut à une ontologie qui refuse d'être une cosmologie, et qui précisément identifie Nature et lumière, au lieu de faire jaillir la lumière des profondeurs de la Nature ? »
} 
dans les connexions de sens qui forment l'armature du monde et sans estomper sa présence impérieuse en devenant l'horizon lointain de référence du savoir objectivant. La nature doit pouvoir émerger dans le monde sans se confondre avec le monde.

En dépit de la rareté relative des analyses qu'il consacre à la question de la nature (qui contraste avec la profusion des références à la thématique du monde naturel et le nombre important des développements autour du concept de physis), il nous semble que la pensée de Jan Patočka permet de constituer un cadre interprétatif à même de contourner le triple écueil d'une nature appauvrie, d'une nature aménagée et $\mathrm{d}^{\prime}$ une nature excédentaire ${ }^{7}$. La conviction qui guide son effort théorique est qu'une élaboration phénoménologique de la nature doit nécessairement prendre son départ au lieu où la nature s'atteste, dans l'expérience que nous en faisons. Pourtant toute expérience se déploie sur fond de monde, au double sens où elle n'est possible que sur le sol du monde et que le monde lui est présent comme l'horizon constant qu'elle co-vise. La question à laquelle la pensée de Patočka nous invite à répondre est donc la suivante : comment la nature apparaît au sein du monde, ou plus précisément, au sein du monde naturel ou du monde de la vie ? La réponse proposée par le philosophe tchèque se laisse saisir à travers deux thèses. La première concerne le statut de la phénoménalité propre à la nature : la nature est captée dans chaque monde naturel comme ce qui s'en excepte, elle se présente comme l'internalisation d'une dimension extérieure et débordante. Autrement dit, alors même que toute exploration de la nature ne peut s'effectuer que sur le sol du monde naturel, la phénoménalité de la nature serait amputée d'une de ses dimensions tant qu'on n'aura pas reconnu le moment non-mondain que celle-ci comporte nécessairement. Cette approche permet de rendre compte de la double dimension- de codage symbolique et d'invariance - qui est propre à l'expérience de la nature. La seconde thèse vise à circonscrire la manifestation la plus

\footnotetext{
${ }^{7}$ Nous nous en tenons dans ce qui suit à la perspective que Patočka développe dans le cadre d'une démarche phénoménologique. On peut toutefois noter que sa réflexion philosophique ne se déploie pas uniquement sur ce plan phénoménologique, qu'il entrevoit même la nécessité d'opérer un dépassement de la phénoménologie au sens strict vers une métaphysique phénoménologique. Dans ce cadre théorique la physis revêtira une nouvelle signification, en ce qu'elle nommera non pas simplement la «nature », mais « le monde au sens fort». Cf. " Méditation sur "Le monde naturel comme problème philosophique" » in Le monde naturel et le mouvement de l'existence humaine (désormais MNMEH), trad. E. Abrams, Dordrecht, Kluwer, 1988, p. 100. Pour une exploration des raisons qui ont conduit Patočka à ouvrir son enquête phénoménologique à des motifs d'ordre métaphysique (ou cosmologique), nous nous permettons de renvoyer à O. Stanciu, « De la manifestation au réel. La phénoménologie asubjective et son autodépassement », Revue de métaphysique et de morale, n 95, 2017/3, p. 303-316.
} 
prégnante de la nature dans le monde naturel (dans tout monde naturel). Elle affirme que l'on peut saisir la phénoménalité la plus saillante de la nature lorsqu'on l'envisage sous la guise de la polarité du ciel et de la terre. Cette thèse - qui a été affirmée avec une force incontestable dans un contexte théorique proche par Klaus Held ${ }^{8}-$ se trouve à l'horizon des développements de Patočka lorsqu'il assimile la terre et le ciel aux «puissances fondamentales du réel »: "seule l'orientation par rapport à la terre et au ciel montre ce qu'est le contact sensible humain - qu'il est possible uniquement dans le mouvement d'un être "du" monde, d'un être qui s'explique non seulement avec les singularités mais aussi avec les puissances fondamentales du réel $»^{9}$.

En explorant le périmètre théorique que ces thèses dessinent nous examinerons successivement 1 ) les axes selon lesquels se déploie l'analyse renouvelée que Patočka donne du monde naturel ; 2) la manière dont le philosophe tchèque se distancie de la compréhension heideggérienne de la nature (telle qu'elle est formulée notamment dans Sein und Zeit et les textes attenants) ; 3) la fonction impartie par Patočka au concept de nature dans son dispositif conceptuel: le passage du monde entendu comme structure préalable de l'expérience au monde au sens fort s'effectue à travers la prise en compte de l'inassimilabilité de la nature au monde naturel.

\section{La redescription du monde naturel}

L'interrogation patočkienne sur la nature est indissociable de son projet de renouvellement de l'analyse du monde naturel. En effet, pour qu'une enquête phénoménologique puisse prendre en charge la question de la nature, il est nécessaire que le monde naturel soit restitué dans toute son ampleur, car «toute phénoménologie est au fond une phénoménologie du monde naturel $\gg^{10}$. Cette orientation assume un double parti pris interprétatif. Premièrement, elle s'appuie sur la thèse selon laquelle la nature ne saurait se manifester que dans le monde, dans la mesure où le monde nomme la dimension d'ouverture au sein de laquelle tout apparaître se trouve inscrit. Ensuite, elle engage une

\footnotetext{
8 Cf. à cet égard K. Held, «Sky and Earth as Invariants of the Life-world » in Phänomenologische Forschungen Sonderband, Phenomenology of interculturality and life-world, E. W. Orth et Chan-Fai Cheung (éd.), Munich, Alber, 1998, p. 22 : «la polarité du ciel et de la terre est un des invariants du monde de la vie humaine. Pourtant cette polarité serait mécomprise et transposée dans un cadre naturaliste si on l'oppose à la culture comme issue de la liberté humaine ».

9 J. Patočka, « Notes sur la préhistoire de la science du mouvement : le monde, la terre, le ciel et le mouvement de la vie humaine », in MNMEH, p. 11.

${ }^{10} \mathrm{~J}$. Patočka, «Le monde naturel et la phénoménologie » in MNMEH, p. 24.
} 
critique de la conceptualité mise en œuvre par la phénoménologie classique pour la saisie du monde naturel qui aurait eu pour conséquence de restreindre de manière indue la place conférée à la nature, en la reléguant aux confins ou dans les limbes du monde.

Lorsqu'il procède à donner une nouvelle description du monde naturel Patočka retrouve et reprend à son compte les acquis majeurs de l'élaboration husserlienne de la Lebenswelt. Le philosophe tchèque insiste, à la suite de Husserl, sur trois caractéristiques positives du monde naturel. Il s'agit en premier lieu d'un domaine de sens préalable qui est investi par nos pratiques et qui nous est directement ouvert « par l'usage de la vie ». Ce monde avec lequel on est uni par une connivence qu'aucun malentendu ne saurait défaire constitue un lieu d'évidences originelles et préthéoriques : "Toutes les disciplines positives et objectives présupposent, antérieurement à elles-mêmes, un monde, dans un certain sens, déjà achevé et fonctionnant, qui ne perd à aucun moment sa validité pour nous tous, un monde préalable qui n'est pas un résultat de l'activité théorique mais, au contraire, la précède ${ }^{11}$. Mais, deuxièmement, le monde est non seulement ce qui soutient en tant que sol toute construction idéelle, mais également ce qui devance en tant qu'horizon l'expérience de la singularité. En ce second sens, le monde de la vie apparaît comme une matrice structurante pour toute expérience : tout contenu d'expérience est inséré dans une trame globale, toute expérience fait fond sur la « donation non perceptive, primaire, préalable $»^{12}$ de la totalité. Saisir une chose $c^{\prime}$ est la ressaisir à partir de ses horizons, c'est donc en faire l'expérience comme d'une chose dans le monde: si " chaque action a son thème, une tâche naturelle qui $\mathrm{m}^{\prime}$ incombe ", " tout thème se transcende de fait, sans qu'il y ait besoin d'y penser ou de se le représenter, vers [une] totalité non thématique », de sorte que " c'est cette totalité qui mérite le titre de monde, de monde naturel de la vie ${ }^{13}$. Enfin, troisièmement, le monde nomme non seulement ce dont nous faisons l'expérience, mais également ce à partir de quoi nous faisons toute expérience. Autrement dit, la Lebenswelt est non seulement le sol où toute production de sens plonge ses racines, mais également le champ où $\mathrm{s}^{\prime}$ accomplit la genèse du sens. Il s'agit donc de faire apparaître non seulement l'irréductibilité du monde de la vie, mais également sa fertilité, sa productivité dans l'ordre du sens.

\footnotetext{
${ }^{11}$ J. Patočka, Introduction à la phénoménologie de Husserl, trad. E. Abrams, Grenoble, Jérôme Millon, 1992, p. 212.

${ }^{12}$ J. Patočka, « Le monde naturel et la phénoménologie » in MNMEH, p. 27.

${ }^{13}$ MNMEH, p. 239.
} 
Bien qu'il adhère à cette triple caractérisation de la Lebenswelt comme sol de l'idéalisation, matrice de l'expérience et patrie du sens, Patočka se démarque radicalement de la manière dont Husserl entend prolonger ces analyses. Le point central de sa critique vise le statut que Husserl attribue au monde de la vie, qui loin d'être reconnu comme un champ autonome, apparaît comme doublement dérivé. En premier lieu, Patočka souligne la dépendance de la thématisation husserlienne du monde de la vie à l'égard du projet de fournir une base assurée aux prestations de sens de la science: "les esquisses présentées sont manifestement orientées sur le problème de l'idéalisation, de l'objectivation, de l'origine des sciences de la nature. Par conséquent, elles relèvent avant tout les traits du monde de la vie naturelle et naïve qui fournissent une base signifiante à l'objectivation et au processus ultérieur de naturalisation ${ }^{14}$. L'association de la question du monde de la vie avec une démarche fondationnelle - la Lebenswelt est indissociable du mouvement régressif de la Rückfrage - est responsable du caractère abstrait des descriptions husserliennes. Ce qui est dévoilé sous ce titre n'est pas le monde de l'expérience dans toute sa richesse structurale : le regard du philosophe ne scrute pas l'horizon de notre vie quotidienne où se nouent nos rapports avec les choses et nos semblables, mais cherche à dégager une instance qui peut fonctionner comme l'assise enfouie de la rationalité scientifique et qui peut procurer une garantie génétique à ses procédures : "La Lebenswelt au sens de Husserl demeure une abstraction régie par la fonction spéciale de la science - la Lebenswelt n'est pas un monde au sens propre ; la conception husserlienne escamote, ne thématise pas, oublie le monde en tant que plan purement phénoménal ${ }^{15}$.

Deuxièmement, Patočka conteste l'orientation d'ensemble de la Krisis qui fait de l'éclaircissement du monde de la vie une entreprise préliminaire, une simple voie détournée pour accéder à la subjectivité transcendantale. En vertu de son adhésion à la thèse selon laquelle toute élucidation de sens demeure insuffisante tant qu'elle ne parvient pas à faire ressortir la prestation (Leistung) subjective qui la soutient, Husserl refuse de faire du monde de la vie un domaine sui generis, structuré par une légalité spécifique. Pour le philosophe allemand, un dévoilement conséquent de la Lebenswelt doit indiquer sa dépendance dans l'ordre de la constitution à l'égard d'une dimension qui lui est externe et le faire ainsi apparaitre comme une formation de sens secondaire. Or, c'est précisément ce pas théorique que Patočka refuse

\footnotetext{
${ }^{14}$ MNMEH, p. 89.

${ }^{15}$ J. Patočka, « Réflexions sur l'Europe », in Liberté et sacrifice, op. cit., p. 212.
} 
de franchir estimant $\mathrm{qu}^{\prime}$ « envisager le monde comme l'accomplissement d'une intersubjectivité fondamentale ${ }^{16}$ équivaut à le «dépouiller de l'indépendance d'être qui caractérise ses "objets", par rapport aux consciences finies, lesquels s'identifient avec leurs substrats corporels ; une métaphysique de l'intersubjectivité transcendantale constituant le monde a besoin de sujets absolus et absolument donnés ${ }^{17}$. La soumission de la Lebenswelt à une exigence gnoséologique et son appréhension dans une optique constitutive auront des effets profonds quant au statut de la nature. Au sein de ce cadre, la nature n'apparaît que comme une dimension constamment mise à distance : soit comme un pôle indéfiniment éloigné que l'ensemble des constructions théoriques cherche à déterminer, mais qui, comme tel, se dérobe à toute expérience ; soit comme une «strate » basale et étanche sur laquelle viennent se greffer les "couches » supérieures de la culture et des valeurs, mais qui, dans sa pureté, reste hors d'atteinte.

Délester l'analyse du monde de la vie du poids que représente cette démarche fondationnelle, arracher ce questionnement de l'orbite transcendantal-subjectiviste sur laquelle Husserl l'a placé conduit Patočka à le saisir dans sa corrélation avec une subjectivité corporelle et active. Le concept qui guide le réaménagement de ce cadre théorique est celui de la praxis, car « le monde de la vie est [...] avant tout le monde de la praxis humaine, le monde où nous mangeons et travaillons, où nous vaquons à des tâches à accomplir par l'intermédiaire $\mathrm{du}$ fonds jamais expressément aperçu mais toujours disponible que représente notre existence physique, corporelle ${ }^{18}$. La Lebenswelt se découvre uniquement à la lumière de notre engagement en son sein, elle n'apparaît comme telle que pour une subjectivité qui ne se trouve pas en position de surplomb, mais qui adhère au monde ${ }^{19}$. En effet, un regard pur, détaché de toute forme d'investissement mondain, ne pourrait que transformer le monde en un grand objet, en une surface plane, sans ombre et mystère, s'étalant devant lui. Or, le dévoilement du monde de la vie n'est possible que si l'on fait apparaître la double dimension d'inhérence et de pouvoir de dévoilement propre à la subjectivité. La promotion de la praxis au rang de pivot théorique de cette démarche est à même de satisfaire à cette double exigence car la praxis permet de rendre compte à la fois de notre appartenance au monde agir c'est s'installer au milieu du monde, de plain-pied avec les cho-

\footnotetext{
${ }^{16}$ MNMEH, p. 228.

17 J. Patočka, « La philosophie de la crise des sciences d'après Edmund Husserl et sa conception d'une phénoménologie du "monde de la vie" » in MNMEH, p. 238.

18 MNMEH, p. 238-239.

${ }^{19}$ Cf. PP, p. 262 : « le monde ne fait pas encontre au moi, le moi est dans le monde avec les choses ».
} 
ses - et de notre pouvoir dévoilant, car toute intervention dans le monde présuppose la saisie des choses à partir d'un réseau de renvois articulé autour de notre agir : apparaître, pour une chose, c'est apparaître « en vue de quelque chose ».

Pourtant, Patočka franchit un pas supplémentaire dans la détermination de la subjectivité en soulignant le lien qui s'établit entre la praxis et le mouvement : « depuis la perception jusqu'à l'activité la plus spirituelle de l'artiste ou à l'attitude méditative du mystique, le faire passe toujours par un mouvement $\gg^{20}$. Une subjectivité située dans le monde, qui assume une localisation au sein du monde, ne se contente pas de saisir des systèmes de renvois dans lesquels les choses se montrent, mais elle accomplit un mouvement vers elles. Par ce mouvement, l'existence se ménage une place parmi les choses, sans pour autant se fixer : si elle devenait une simple présence, une chose parmi les choses, elle ne pourrait plus faire apparaître le monde. La subjectivité peut donc être caractérisée par sa mobilité ou, plus précisément, par son auto-mobilité. Le mouvement du soi dit appartenance au monde sans enlisement dans la positivité, pouvoir dévoilant sans position de survol ${ }^{21}$.

Dans la mesure où la Lebenswelt est située dans une corrélation indéfectible avec la subjectivité et pour autant que la subjectivité ait été ressaisie comme mouvement, il s'ensuit que le monde de la vie doit être conçu comme l'articulation des horizons dans lesquels se déploie notre mobilité. Comme le note Patočka, « le monde naturel est le monde du mouvement; sa clef est à trouver dans le mouvement au monde, le mouvement d'un être "du" monde $»^{22}$. La redéfinition du monde naturel comme monde du mouvement-monde au sein duquel se déploie le mouvement propre à l'existence et monde qui est articulé par ce mouvement - nous semble être le pas décisif franchi par Patočka en vue d'une compréhension renouvelée de la nature. La conviction qui anime ses développements est que la voie menant à une élucidation phénoménologique de la nature est indissociable de la question des invariants phénoménaux du mouvement de l'existence. La nature apparaîtra ainsi comme ce qui fournit un cadre pour le mouvement de l'existence, des repères invariants par rapport auxquels elle peut se situer, les témoins devant lesquels elle peut déployer son libre périple. En effet, l'attestation du mouvement présuppose la fixité d'un repère.

\footnotetext{
${ }^{20}$ PP, p. 70.

${ }^{21}$ Pour un exposé détaillé de l'interprétation patočkienne de la subjectivité comme mouvement, nous renvoyons à R. Barbaras, L'ouverture du monde. Lecture de Jan Patočka (Chatou, La Transparence, 2011) et notamment à la section intitulée « Existence corporelle et mouvement ».

${ }_{22}$ J. Patočka, « Le monde naturel et la phénoménologie » in MNMEH, p. 47.
} 
Comme Patočka le note dans un texte de 1967 : « Le monde naturel [...] est une totalité dans laquelle nous effectuons nous-mêmes le mouvement de notre vie qui, en tant que mouvement au sein de la totalité, est rapporté à cette totalité [...] ; les puissances au sein desquelles il se déploie en sont les repères $\gg^{23}$. Dans le même texte, il donne une détermination plus précise à ces " puissances » en insistant d'abord, à la suite de Husserl et de Heidegger, sur l'importance d'octroyer un statut phénoménologique à la Terre : «Le repère propre au flux réalisateur de notre activité effectuante (qui est mouvement au sens le plus originel du terme) est un substrat permanent et immobile - la Terre $»^{24}$. Pourtant, à la différence de ses maîtres fribourgeois, Patočka soutient la nécessité d'ajouter un repère supplémentaire, et de faire du Ciel un problème philosophique (envisagé comme un des repères du mouvement de l'existence) : " Il y a cependant encore un autre repère, essentiellement lointain, impalpable, dont on ne peut se rendre maître par le moyen du toucher corporel [...] : les cieux, la lumière, les lumières et les "corps" célestes, tout ce qui ferme notre horizon sans clôture, ce qui forme le dehors comme un dedans qui nous enferme à chaque instant $»^{25}$. La terre, l'assise du mouvement de l'existence, et le ciel, son pôle possibilisant, forment les entours du champ de sens dans lequel évolue l'existence humaine. La terre et le ciel sont des invariants phénoménaux : ils participent à la configuration du monde non pas en tant que factualité brute, mais en tant qu'ils apparaissent dans le monde (ils ne relèvent donc pas d'une nature au sens naturaliste). Pourtant, cette apparition n'est pas à son tour quelque chose qui se laisse moduler par la dynamique de l'existence, qui pourrait être incorporée sans reste dans le monde.

Le monde naturel apparaît ainsi comme une constellation de lieux articulés autour d'un axe dynamique - le mouvement de l'existence. Pourtant, le mouvement de l'existence est soumis à une certaine configuration qui encadre par avance les variations auxquelles il peut donner lieu. Il est constamment pris dans une situation signifiante dont il n'a pas dessiné les coordonnées, il doit s'inscrire dans une dimension qui résiste à l'appropriation. C'est cette dimension qui doit être appelée la nature. La nouvelle modulation que Patočka donne à la problématique du monde naturel permet de l'affranchir de sa dépendance à l'égard d'un horizon uniquement anthropologique et de l'installer

\footnotetext{
23 Ibid., p. 46.

24 Ibid., p. 30.

25 Ibid., p. 32.
} 
dans la densité (inappropriable, mais pourtant phénoménale) d'une nature.

\title{
II. Expérience de la nature et comportement démondanéisant
}

La caractérisation de la nature comme l'ensemble des "puissances fondamentales du réel » doit être étoffée et approfondie à travers l'indication des contextes phénoménaux qu'elle est à même d'éclairer. Patočka s'acquitte de cette tâche dans un passage décisif tiré d'un manuscrit de travail du début des années soixante-dix intitulé « Forme du monde de l'expérience et expérience du monde » (Weltform der Erfahrung und Erfahrung der Welt) que nous citerons in extenso :

\begin{abstract}
C'est seulement dans l'«environnement humain » qu'il y a des chaises; la "nature » ne connaît rien de la sorte. Pourtant, l'on fait aussi l'expérience de la nature dans le travail et l'être-en-chemin, dans la nécessité d'y trouver justement son chemin, un gîte, le boire et le manger. La nature toujours présente, c'est le jour et la nuit, la terre et le ciel. Le jour et la nuit se rapportent à quelque chose d'indépendant des hommes, quelque chose cependant qui, dans la différence qui luit originairement, n'est pas immédiatement co-éclairci et dont la clarification se poursuit à l'infini dans les sciences de la nature avec l'analyse de la nature en général; [...] En lisant chez Heidegger les analyses du monde ambiant de la quotidienneté, l'on a parfois l'impression que la nature serait un simple concept-limite, présente uniquement sous les espèces du matériel destiné à l'ouvrage. Pourtant, la nuit et le jour, la terre, le ciel et les "éléments" sont également là dans le monde de l'ouvrage journalier, de même que l'arbre couvert de poussière que l'on remarque à peine en bordure d'une rue animée. Cela dit, "la nature » a le trait particulier de présenter le jour et la nuit, la terre et le ciel en une guise non morcelée et non rassemblée. La nature n'est pas là uniquement ou au premier chef comme carrière, forêt, etc., mais en tant que cadre non thématique et changeant. L'on "utilise " la nature non seulement lorsqu'on extrait du matériel pour les choses de notre usage, la nourriture, etc., mais également en "étant naturellement", en entendant, en voyant, en se laissant pénétrer par sa clarté ou en étant sensible à l'absence de celle-ci ${ }^{26}$.
\end{abstract}

La thèse centrale qui ressort de ce passage est qu'il y a bel et bien une expérience de la nature comme nature. Pourtant, soutenir cette thèse ne revient pas à nier que le terrain sur lequel cette expérience s'effectue est celui du monde naturel. En effet, l'expérience de la nature est indissociable de notre engagement dans le monde, elle ne se dévoile

\footnotetext{
26 J. Patočka, Weltform der Erfahrung und Erfahrung der Welt in Vom Erscheinen als solchem. Texte aus dem Nachlass, H. Blaschek-Hahn et K. Novotný (éd.), Fribourg, Karl Alber, 2000, p. 102-103; « Forme du monde de l'expérience et expérience du monde » in PP, p. 213.
} 
qu'à l'horizon des mouvements que notre existence réalise. En évoquant ces mouvements, Patočka se réfère non seulement à l'activité par laquelle nous aménageons le monde, en le rendant conforme à nos besoins, mais aussi aux différentes déclinaisons de notre manière d'habiter le monde, à travers « le travail et l'être-en-chemin, la nécessité d'y trouver justement son chemin, un gîte, le boire et le manger ». Ce complexe de questions est analysé selon deux axes. En premier lieu, Patočka tente, à la faveur d'une démarche critique, de montrer que Heidegger n'a pas suffisamment pris en compte la diversité de notre engagement dans le monde - la pluridirectionnalité du mouvement de l'existence - en référant la nature à l'expérience que nous en faisons dans cet engagement particulier qu'est l'utilisation instrumentale. Deuxièmement, le philosophe tchèque esquisse sa propre réponse à la question de la phénoménalité de la nature, en privilégiant la référence aux repères du mouvement de l'existence - la terre et le ciel.

Afin de mesurer le sens et l'ampleur de la critique que Patočka adresse à la thématisation heideggérienne de la nature, il est nécessaire de restituer brièvement la position défendue par le philosophe allemand. Les analyses que Heidegger consacre à la question de la nature dans Sein und Zeit et dans les cours qui gravitent autour de cette œuvre prennent place au sein du débat qu'il engage avec la compréhension traditionnelle du monde comme totalité additive de l'étant. La tâche qu'il assigne à sa démarche est celle de défaire l'assimilation, structurante pour l'ontologie traditionnelle, entre la nature et le monde, et de montrer qu'avant de nommer l'ensemble des étants que nous pouvons appréhender, le monde désigne ce «dans quoi », ce «à l'intérieur de quoi » (das Worin) cette appréhension peut se réaliser, le champ de l'apparaître qui fournit à chaque étant la possibilité de venir à la lumière. En comprenant le monde comme une simple totalité additive, dépourvue de toute référence à l'apparaître et à la subjectivité, l'ontologie traditionnelle " escamote » le phénomène du monde et fait passer sous ce nom ce qui convient davantage d'être appelé nature : " quand bien même elle réussirait à fournir l'explication la plus pure de l'être de la nature, en parfaite conformité aux propositions fondamentales qu'énonce la science mathématique de la nature sur ce type d'étant, cette ontologie ne saurait atteindre le phénomène du "monde". Car la nature est elle-même un étant qui fait encontre à l'intérieur du monde $»^{27}$. Entre le monde et la nature, la distance est infranchissable :

${ }^{27}$ GA 2, p. 86 ; trad. p. 71. 
« La nature n'est pas identique au monde. La nature est présence-subsistante, alors que le monde existe $»^{28}$.

Pourtant, Heidegger ne se contente pas de dénoncer la confusion entre monde et nature et de souligner l'antériorité de droit du monde par rapport à la nature, mais insiste également sur l'exiguïté du concept de nature développé par l'ontologie traditionnelle. Ce concept est non seulement dépendant d'un certain comportement démondanéisant du Dasein - l'attitude théorique - mais, en outre, il est orienté d'avance sur les exigences émanant du dispositif des sciences naturelles - pour lesquelles la nature nomme une multiplicité d'occurrences extérieures les unes aux autres et rattachées uniquement par la causalité. Pourtant, la nature peut apparaître dans le monde autrement que comme «l'être-sous-la-main du sous-la-main en général ${ }^{29}$ ou comme la totalité de la Vorhandensein. Heidegger tente de faire droit à une expérience de la nature qui a le monde comme sol de son déploiement, mais qui n'est pas pré-ordonnée à la science mathématisée. Comme l'atteste un célèbre passage du $\S 15$ d'Être et temps : "Dans l'outil dont on se sert et par le fait de s'en servir est co-découverte la nature" - la "nature" telle qu'éclairée par les produits naturels [...] La forêt est réserve de bois, la montagne est carrière de pierre, la rivière est force hydraulique, le vent est vent "dans les voiles". Avec la découverte $\mathrm{du}$ " monde ambiant » vient à notre encontre une "nature" ainsi découverte $»^{30}$.

Il y a donc une expérience de la nature qui ne relève pas d'un processus démondanéisant : ceci représente le point d'accord fondamental entre la position de Heidegger et celle de Patočka. Plus encore, il faut souligner que la nature ne se dévoile qu'à l'aune de la préoccupation, il ne peut apparaître qu'au sein de notre engagement dans le monde. Si Patočka pourrait bien souscrire à la thèse selon laquelle la nature ne se découvre que sur la base de notre comportement praxique, il rejette la restriction tacite que Heidegger fait subir à la praxis en $l^{\prime}$ assimilant à $l^{\prime}$ « utilisation ». Une telle restriction entraîne deux conséquences : en premier lieu, elle conduit à la méconnaissance de la complexité et de la différenciation interne des horizons dans lesquels la

\footnotetext{
${ }^{28}$ M. Heidegger, Geschichte der Philosophie von Thomas von Aquin bis Kant, GA 23, H. Vetter (éd.), 2006, Francfort-sur-le-Main, Klostermman, p. 18.

${ }^{29}$ M. Heidegger, Vom Wesen der menschlichen Freiheit. Einleitung in die Philosophie, GA 31, H. Tietjen (éd.), Klostermann, Francfort-sur-le-Main, 1982 ; trad. E. Martineau, Paris, Gallimard, 1987, p. 184.

${ }^{30}$ GA 2, p. 70 ; trad. p. $72-73$.
} 
praxis se déploie ${ }^{31}$; deuxièmement, elle conduit à un appauvrissement du sens de la nature, qui devient " un simple concept-limite, présente uniquement sous les espèces du matériel destiné à l'ouvrage ».

$\mathrm{Si}$ le complexe de renvois qui détermine la configuration du monde est toujours articulé autour d'un " agir » vivant - ou plutôt d'un mouvement dévoilant - il serait hâtif de supposer que le seul champ dans lequel l'agir peut se déployer est celui de la manipulation des outils. En effet, affirmer le caractère engagé et pratique de notre rapport au monde n'équivaut pas à soutenir qu'il est d'ordre instrumental. Or, la critique de Patočka vise précisément le caractère hégémonique de l'usage instrumental, le fait que le commerce avec les ustensiles masque d'autres formes $d^{\prime}$ " usage de la vie » :

\begin{abstract}
La question se pose de savoir à quoi rattacher, dans les analyses du temps présentées par Heidegger dans Sein und Zeit, les comportements humains "ouverts » qui, tels les actes de dévotion, les activités de l'artiste ou du penseur, ne soit ni une manipulation de pragmata ni un comportement de sollicitude envers des personnes. Ces comportements concourent à former notre environnement, évoluent sans nul doute dans le cadre du souci temporel, dont la présence aux choses représente une dimension indispensable. Pour autant, ils n'ont en vue ni un hou heneka immédiatement compréhensible dans la sphère de la quotidienneté, ni une action historique résolue capable de laisser son empreinte sur les masses humaines pour une période plus ou moins longue. Or, Sein und Zeit n'envisage expressément que ces deux mouvements [...], mouvements qui débouchent, l'un et l'autre, sur l'étant mondain, sur l'engagement dans l'étant mondain ${ }^{32}$.
\end{abstract}

Il est vrai, comme Patočka ne manque pas de le souligner, que l'intervention historique du Dasein résolu ou la sollicitude à l'égard d'autrui - phénomènes thématisés par Heidegger - ne sauraient être situées dans le champ de l'usage instrumental. Néanmoins, il demeure que le point de référence de ces comportements se situe toujours dans

\footnotetext{
${ }^{31}$ Cette même ambiguïté qui a été mise en évidence à l'égard de l'analyse du monde ambiant par Claude Romano qui note que Heidegger «opère [...] au $\S 15$ un glissement sémantique significatif. Heidegger part d'une analyse du Zeug en termes d'usage (Umgang) - terme dont la signification est ample et peut être rendue également par "commerce", "fréquentation" - pour aussitôt substituer à cette notion celle de Gebrauch, utilisation, voire celle de Zeughaftigkeit, ustensilité. Mais de l'usage du monde en un sens augustinien à l'utilisation et à l'ustensilité il y a un pas, et la transition d'un concept à l'autre n'est ni explicite ni vraiment justifiée par Heidegger. Or, beaucoup de choses que nous comprenons prélinguistiquement et revêtent pour nous un sens à la lumière de nos possibles pratiques ne peuvent pas être appréhendées en termes de renvois fonctionnels à autre chose : nous comprenons des actions, des rites, des gestes délibérés ou indélibérés, des expressions faciales, des œuvres d'art, des jeux, et ainsi de suite », (C. Romano, $A u$ cour de la raison, la phénoménologie, Paris, Gallimard, 2010, p. 688).

${ }^{32}$ J. Patočka, Le monde naturel comme problème philosophique (désormais MNPP), trad. E. Abrams, Paris, Vrin, 2016, p. 254.
} 
le domaine de l'intramondain et que Heidegger n'indique pas la manière dont la nature pourrait $s^{\prime}$ annoncer au sein de ces comportements. La thèse implicite avancée par Patočka est qu'une saisie phénoménologique de la nature présuppose la mise au jour des mouvements qui nous portent non pas vers des singularités qui apparaissent au sein du monde, mais vers les domaines plus amples qui représentent les coordonnées de déploiement du monde naturel.

\section{Les repères du mouvement de l'existence}

Toute action humaine se dépasse vers l'horizon total du monde. Or si Patočka peut encore admettre que cette totalité renvoie à l'existence humaine - pour autant que le monde naturel est le corrélat du mouvement de l'existence - il rejette l'idée selon laquelle l'articulation d'ensemble du monde gravite autour de cet « en-vue-de-quoi » qu'est le Dasein. L'ajointement interne et la syntaxe du monde de la vie ne sont pas fixés par le mouvement de l'existence. C'est dans ce contexte que l'évocation patočkienne de la polarité fondamentale du ciel et de la terre reçoit son sens plein. En effet,

notre explication avec les choses intramondaines a lieu sur le fond de la terre et du ciel-arrière-plan qui, bien sûr, n'est pas essentiellement ni toujours nécessairement thématisé. La terre et le ciel n'entrent jamais pleinement dans le cadre du comportement pratique et de l'attitude correspondante. La terre et le ciel sont, certes, des repères pratiques, mais ils ne sont pas que cela: ils ont une profondeur propre que nous ressentons à tout instant, encore qu'elle ne soit pas tournée vers nous ${ }^{33}$.

On le voit, penser la terre et le ciel revient à relever le mouvement par lequel des dimensions externes au monde naturel sont devenues inhérentes à celui-ci tout en gardant encore une face qui "n'est pas tournée vers nous ». Que la terre est le ciel soient des repères pratiques, mais «qu'ils ne soient pas seulement cela » témoigne précisément du statut phénoménologique particulier que la nature possède pour Patočka: la présence de la nature dans le monde naturel relève de l'absorption de ce qui se retient, de la capture de ce qui est (et demeure) hors-champ.

Pourtant, l'élucidation de la teneur propre de la polarité ciel-terre ne peut pas rester à l'indication de la prise oblique qu'elle appelle. Une enquête supplémentaire devrait pouvoir indiquer la manière dont elle

${ }_{33}^{3}$ J. Patočka, « Le monde naturel et la phénoménologie » in MNMEH, p. 32. 
intervient dans la configuration du monde naturel. À suivre Patočka, la terre présente un triple visage. En premier lieu, notre mouvement ne saurait être assimilé à un déplacement à l'égard des choses, car tout déplacement présuppose une adhérence préalable à la terre. Ainsi, la terre nomme le repère horizontal du mouvement de l'existence, « un appui solide, le substrat de tout mouvement, aussi bien le nôtre que celui des choses $»^{34}$. En effet, « le mouvement se déploie quelque part. Ce quelque part n'est pas une place dans l'espace objectif mais le sol, le point d'ancrage pour toutes les places possibles - là où repose non pas mon corps mais mon mouvement, le sol qu'il foule toujours, même si ce sol est un vaisseau spatial ou un nuage $»^{35}$. Deuxièmement, la terre est envisagée en tant que puissance et mise en rapport avec la dimension vitale de la subjectivité : « le règne de la terre est en même temps, dans la sphère de la vie, un pouvoir sur la vie et la mort, car la terre n'est pas seulement ce qui soutient et qui porte, mais encore celle qui nourrit $»^{36}$. Selon cette optique, la terre soutient le mouvement de l'existence non pas en vertu de sa solidité et de sa fermeté, mais bien plutôt en vertu de sa fécondité et de sa profusion, en tant qu'elle est une source intarissable et un sol nourricier. Enfin, comme le mouvement de notre existence est une avancée dévoilante et pour autant que l'apparaître est toujours configuré selon la polarité de l'ouvert et du retrait, la terre apparaîtra comme ce qui se « réserve » dans toute manifestation, la dimension compacte dans laquelle tout sens s'engloutit.

$\mathrm{Au}$ triple visage de la terre (repère horizontal du mouvement, sol nourricier et retrait affectant tout apparaître) correspond une triple appréhension du ciel. Considéré eu égard à notre mobilité, le ciel apparaît comme "essentiellement lointain", comme le donateur de la distance, comme ce qui suscite l'essor du mouvement, sa relance continue, « le corrélat mondain de notre pouvoir d'arrachement moteur ${ }^{37}$. Eu égard à la dimension vitale de notre existence, le ciel apparaît comme ce qui rythme notre mouvement vital « en tant que s'y relaient le jour et la nuit, la lumière et les ténèbres avec tous leurs cycles, leurs avènements et leurs évolutions ${ }^{38}$. Enfin, en regard de l'articulation interne de l'apparaître, le ciel nomme l'espace de la clarté et le principe de différenciation : les choses peuvent apparaître uniquement dans la clarté du ciel qui ouvre la distance, qui creuse un écart entre elles, qui les détachent les unes des autres.

\footnotetext{
${ }^{34}$ MNMEH, p. 7.

${ }^{35}$ R. Barbaras, L'ouverture du monde, op. cit., p. 192.

${ }^{36} \mathrm{MNMEH}$, p. 7.

${ }^{37}$ R. Barbaras, L'ouverture du monde, op. cit., p. 195.

${ }^{38} \mathrm{MNMEH}$, p. 8.
} 
Les caractéristiques que Patočka attribue à la polarité terre-ciel nous permettent d'y reconnaître une dualité qui relève de la nature, c'est-àdire comme appartenant à ce qui se présente nécessairement dans chaque monde naturel tout en le débordant. Le ciel et la terre sont simultanément des points d'appui de la phénoménalité et des points de retrait : ils participent à la configuration du champ de l'apparaître, sans avoir leur source dans un accomplissement subjectif; ils ont une portée structurante dans l'ordre du sens, sans que pour autant leur teneur propre puisse être épuisée lorsqu'elle est envisagée dans l'orbe du sens ; ils représentent une structure non-relative mais qui est codée symboliquement chaque fois de façon différente.

L'aspiration qui traverse l'ensemble des analyses que Patočka consacre à la question de la nature est celle de déterminer le relief singulier que revêt sa phénoménalité, la place particulière qu'elle occupe au sein du monde naturel. Comme nous l'avons indiqué, c'est en proposant un élargissement et un approfondissement du sens du monde naturel que Patočka se donne les moyens pour parvenir à une saisie de la nature comme phénomène. En assumant cette orientation, il ne s'agit pas de faire de la nature la force qui propulse l'apparaître, mais plutôt de reconnaître en elle une de ses dimensions. Autrement dit, la nature n'est pas l'autre du monde, l'envers du décor, mais elle désigne un des axes d'organisation de la scène de l'apparaître. La nature que la phénoménologie explore n'est pas une nature pré-mondaine, voire cosmogène, mais une nature déjà mondiale (sans qu'elle soit pour autant intramondaine). C'est cette difficulté que Patočka affronte de façon directe : ne pas exclure la nature du monde, sans en faire un moment dérivé de celui-ci.

La nature n'existe qu'inscrite dans une constellation de sens, dans un monde naturel. En effet, chaque monde naturel a « sa » nature. Mais la nature ainsi " appropriée » ne saurait être réduite à sa " face tournée vers nous ». Cette situation théorique, qui peut être décrite à travers des formules telles que « la captation de ce qui s'excepte » ou « l'internalisation d'une dimension débordante » nous permet de rendre compte de la tension qui traverse tout monde naturel : entre l'ordre manifeste qui affirme sans cesse ses ambitions hégémoniques en cherchant à inscrire dans son territoire idiomatique tout ce qui lui fait face et l'assise sur laquelle il s'édifie qui, tout en participant à la configuration du monde, ne se laisse pas résorber en lui. Mais en outre, l'irréductibilité de la nature au monde naturel est ce qui nous empêche de prendre une certaine configuration globale de l'expérience (un monde naturel) pour le monde lui-même. Ainsi, la nature fait apparaître le caractère non-autarcique du monde naturel, son absence d'au- 
tonomie ; il y a du sens qui n'est pas notre œuvre, qui n'a pas sa source dans nos projets de sens et leurs sédimentations. Que le monde naturel n'ait pas sa tenue en lui-même, qu'il ne puisse pas se soutenir luimême, qu'il doive composer sans cesse avec une dimension qui le déborde, tels nous semble être les acquis majeurs de l'exploration patočkienne de la nature ${ }^{39}$.

${ }^{39}$ Cet article a été rédigé dans le cadre du projet de recherche "Finitude and Meaning. Phenomenological Perspectives on History in the Light of the Paul Ricoeur - Jan Patočka Relationship" (code du projet: PN-III-P1-1.1-TE-2016-2224), soutenu par UEFISCDI et réalisé auprès de l'Institut de Philosophie « Alexandru Dragomir » (Société Roumaine de Phénoménologie). 